\title{
Direct quantification of protein glycan phosphorylation
}

\author{
Stephanie A. Ketcham, Muhammad Ashraf, and Chikkathur N. Madhavarao \\ Division of Product Quality Research, Office of Testing and Research, Office of Pharmaceutical Quality, \\ Center for Drug Evaluation and Research (CDER), Food and Drug Administration (FDA), Silver Spring, MD
}

BioTechniques 63:117-123 (September 2017) doi 10.2144/000114587

Keywords: Iysosomal enzymes; phosphorylation; phospho-glycans; glycosylation; mannose-6-phosphate

Phosphorylation is an important post-translational modification (PTM) of proteins and a critical quality attribute for protein therapeutics, especially if it is required for protein function or sub-cellular targeting. Most current methods to quantify phosphorylation are time-consuming, indirect, or require specific instrumentation and technical skills. Here, we report the adaptation of a phosphatespecific binding dye and common laboratory instruments for quantification of relative amounts of phosphorylated glycans as well as phosphorylation of amino acid residues on the backbones of proteins. Our results show that quantification of phosphorylation using the new method agrees with published data on the number of phosphorylated glycosylation sites for two lysosomal enzymes: $\beta$ glucuronidase (GUS) and cathepsin D.

Post-translational modifications (PTM) are often essential for protein function and proper sub-cellular localization. Phosphorylation of amino acid (i.e., serine, threonine, and tyrosine) sidechains is essential for activating and deactivating protein kinases and phosphatases to regulate the cell cycle and many other cellular processes (1-3). In addition to phosphorylation regulating protein function, glycosylation of proteins can help with proper protein folding and stability as well as affect protein function $(4,5)$. Furthermore, within the secretory pathway, glycan modifications often act as "zip codes" to localize proteins to various organelles. One such example is the addition of mannose-6-phosphate (M6P) to proteins containing high-mannose glycosylation $(6,7)$, a phosphorylation event that occurs within the cis-Golgi and results in targeting of the M6P-containing protein to the lysosome.

Lysosomes are the sub-cellular organelles where most metabolic waste clearance occurs through the action of acid hydrolases. Deficiency in the function of any one of these lysosomal enzymes leads to accumulation of a specific metabolic waste that is normally hydrolyzed and cleared by that specific enzyme (8-10). This accumulation of waste leads to large vacuole formation in the cells, resulting in $>50$ known metabolic syndromes referred to as Iysosomal storage disorders (LSDs) (11). This buildup of waste can lead to several clinical presentations that involve entire organ systems, generally presenting with muscular, respiratory, and neurological defects, among others (11). Development of such a disorder is seen in nearly 1 in 8000 live births, with symptoms presenting months to years after birth (12). Some LSDs are treated with functional enzyme replacement therapies (ERTs), which are approved by the FDA. Glycosylation with a phosphorylated mannose is critical for the efficacy and potency of many of these ERT enzymes, since they are targeted to lysosomes via the M6P receptors $(6,7)$. Therefore, the level of M6P content in these enzymes is a critical quality attribute (CQA) that needs to be assessed. However, current methods to quantify this PTM require specialized equipment and technical skills, as in the case of the most commonly used technique, mass spectrometry. Here, we present two procedures to examine phosphorylation of both amino acid side-chains and glycans utilizing common laboratory equipment and a phosphate-specific binding dye (13). Furthermore, we show that it is possible to distinguish if the phosphate is present on the side-chain of an amino acid residue or a glycan. We demonstrate the utility of these methods in assessing the relative amounts of phosphorylated glycans in human cathepsin D and purified recombinant human $\beta$-glucuronidase (GUS), which was produced by a $\mathrm{CHO}-\mathrm{K} 1$ cell line, both of which are lysosomal enzymes containing at least one M6P $(14,15)$.

\section{METHOD SUMMARY}

Relative amounts of protein phosphate moieties are quantified by sequential staining of SDS-PAGE-separated proteins using a phosphate-specific binding dye and a protein-specific binding dye. In addition, samples are pretreated with either endoglycosidase or phosphatase to identify glycan-bound or amino acid sidechain-bound phosphates. 


\section{Materials and methods}

Reagents

Recombinant human GUS was produced from a $\mathrm{CHO}-\mathrm{K} 1$ cell line as reported previously (16). Unpurified GUS from the spent media was either dialyzed against buffer (10 mM Tris-HCl, pH 8.0, with 10\% glycerol) or purified as described previously (17). Cathepsin D (21940130U; Fisher Scientific, Pittsburgh, PA), peptide$\mathrm{N}$-glycosidase F (PNGase F) (P0704; New England Biolabs, Ipswich, MA) and alkaline phosphatase (10108146001; Sigma Aldrich, St. Louis, MO) were obtained from the respective manufacturers. For SDS-PAGE, Criterion TGX precast gels (5671094 or 5671095; Bio-Rad, Hercules, CA) were used. The following specific stains were purchased: Phos-Tag Phosphoprotein Gel Stain (P005A and P005B; GeneCopoeia, Rockville, MD), eLuminol Protein Gel Stain (P003A, GeneCopoeia), and PageBlue Protein Staining Solution (24620; Thermo Scientific, Waltham, MA). All buffers and aqueous reagents were prepared using deionized and filtered Milli-Q water (specific resistance: $18.2 \mathrm{M} \Omega / \mathrm{cm}$ ).

\section{SDS-PAGE analysis of}

\section{phosphate content}

For SDS-PAGE phosphate quantification we have adopted three specific stains (i): Phos-Tag for specifically staining phosphate moieties, detected by fluorescence (excitation/emission: 550/580 nm); (ii) eLuminol for staining total proteins, detected by fluorescence (excitation/emission: 460/600 nm); and (iii) PageBlue for determining relative mobility of the treated and untreated proteins, detected by white light. We quantified total protein with eLuminol stain, as it binds to the backbone of the polypeptides rather than specific side chains as does PageBlue. However, eLuminol does not bind well to pre-stained molecular weight standards because the backbone has been modified, preventing detection and assessment of relative mobility of the standards, which necessitated adoption of PageBlue for that purpose.

To determine the average number of phosphate groups present on GUS or cathepsin D, proteins were separated by SDS-PAGE on a $4 \%-20 \%$ TGX precast gel, along with ovalbumin standards (1-15 $\mu g)$. Each SDS-PAGE gel contained four or more ovalbumin concentrations to

A

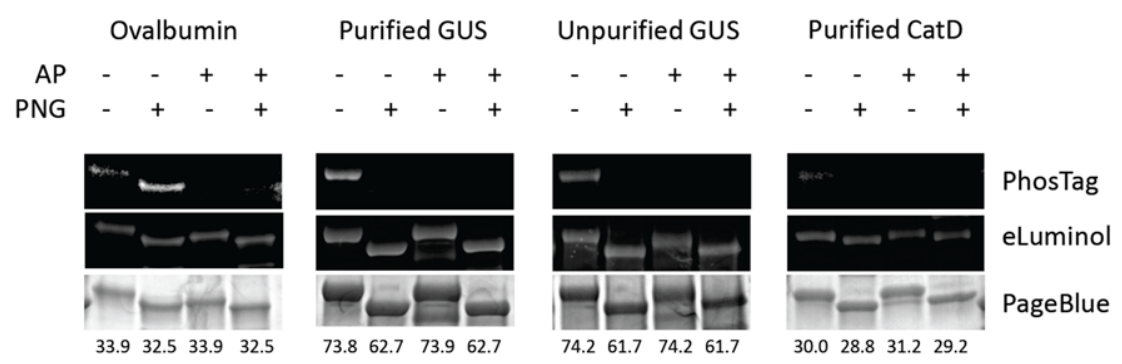

B

\section{eLuminol ${ }^{\mathrm{TM}}$ Stained Standards}

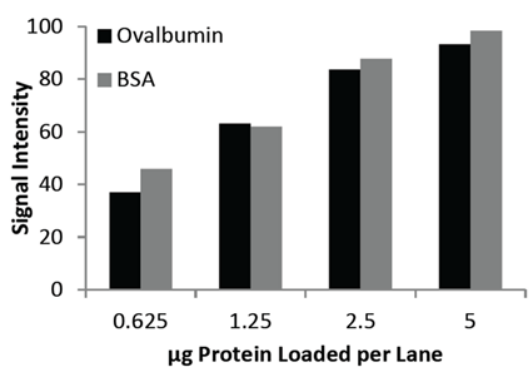

D

\begin{tabular}{|c|c|c|}
\hline Sample & Number of Phosphosites & Standard Error \\
\hline Purified GUS & 1.71 & 0.13 \\
\hline Unpurified GUS & 1.98 & 0.29 \\
\hline Purified CatD & 1.42 & 0.47 \\
\hline
\end{tabular}

Figure 1. Determination of phosphate content by SDS-PAGE. (A) SDS-polyacrylamide gels stained with PhosTag (top), eLuminol (middle), and PageBlue (bottom) resolving control samples or samples treated with alkaline phosphatase (AP) and/or peptide-N-glycosidase F (PNGase F; PNG). The calculated molecular weights of the treated and untreated samples are indicated below the PageBlue-stained gel. (B,C) Signal intensity for a dilution series of ovalbumin resolved with SDS-PAGE and stained with eLuminol (B) and Phos-Tag (C). (D) Quantification of the average number of phosphorylation sites per molecule ( $n>7$ for each sample).

determine the average signal intensity proportional to the amount of protein, which was used in the calculations. For staining with Phos-Tag, eLuminol, and PageBlue, we followed manufacturers' protocols. The TGX precast gel was first stained with Phos-Tag Phosphoprotein Gel Stain (Phos-Tag) and imaged with an E-Gel Imager System using a UV light base (Invitrogen, Carlsbad, CA) (Figure $1 A$, top). The gel was then stained with eLuminol Protein Gel Stain and imaged with an E-Gel Imager System using a blue light base (Invitrogen) (Figure 1A, middle). Finally, the gel was stained with PageBlue Protein Staining Solution and imaged using a blue light base and a white light adaptor (Figure 1A, bottom).

All image analyses were performed with GelQuant Express Analysis Software (Invitrogen). The band intensities of the ovalbumin samples were independently measured from both the Phos-Tag and eLuminol stains and then used to determine the proportionality of the signal intensity per microgram of protein or phosphoprotein (Figure 1B). The Phos-Tag intensities of the ovalbumin standards were used to calculate the average intensity proportional to the amount of protein: (F2-F1)/(P2-P1), where $F$ is fluorescence intensity and $P$ is the amount of protein. Given that ovalbumin has two phosphorylation sites (18-20), the measured Phos-Tag band intensities were divided by two in order to determine the signal intensity per phosphorylation site (Figure 1C). The intensities per microgram of protein or phosphoprotein calculated with the ovalbumin standards were used to determine the amount of total protein and phosphoprotein for GUS and cathepsin D. The intensities falling within the linear range were used for quantifi- 
A

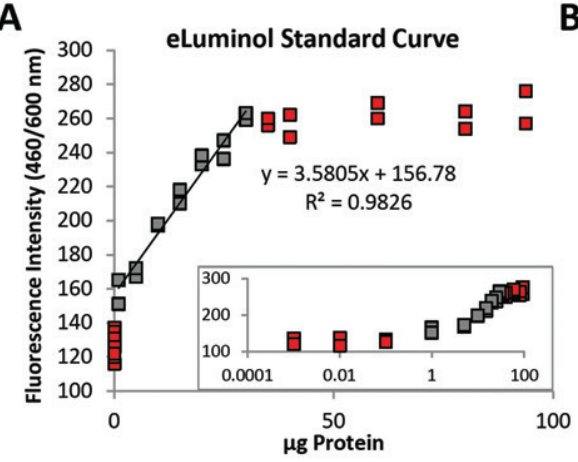

B

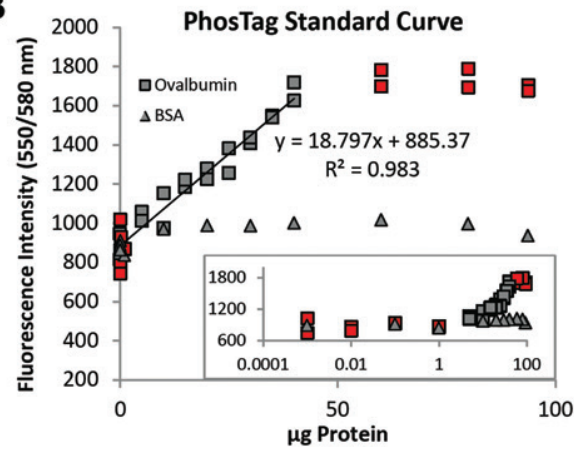

C

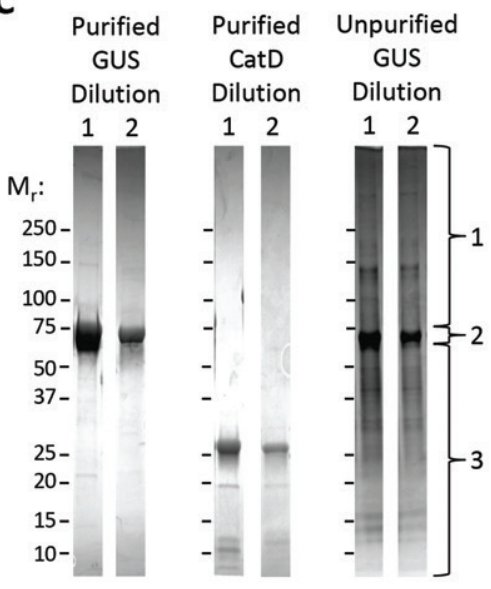

D

\begin{tabular}{|c|c|c|c|}
\hline & Section & $\begin{array}{c}\text { Integrated } \\
\text { Intensity }\end{array}$ & $\begin{array}{c}\text { Purity } \\
\text { Percentage }\end{array}$ \\
\hline \multirow{4}{*}{$\begin{array}{c}\text { Dilution } \\
1\end{array}$} & Section 1 & 14464754 & \multirow{2}{*}{$42 \%$} \\
\cline { 2 - 3 } & Section 2 & 22379531 & \multirow{2}{*}{4} \\
\cline { 2 - 3 } & Section 3 & 16184842 & \\
\hline & \multicolumn{2}{|c|}{} \\
\hline \multirow{2}{*}{$\begin{array}{c}\text { Dilution } \\
2\end{array}$} & Section 1 & 14516203 & \multirow{2}{*}{$43 \%$} \\
\cline { 2 - 3 } & Section 2 & 19648869 & \\
\cline { 2 - 3 } & Section 3 & 11425627 & \\
\hline
\end{tabular}

Figure 2. Determination of phosphate content by immobilization in a 96-well plate. Standard curves generated from a dilution series of ovalbumin immobilized in $2 \%$ agarose and stained with eLuminol (A) and Phos-Tag (B). Red data points were excluded from the standard curve, as they are not within the linear range of detection. The insets in (A) and (B) present the standard curves plotted on a log scale to show the lower threshold of the linear range of detection. (C) Two dilutions of the purified $\beta$-glucuronidase (GUS), purified cathepsin D (CatD) and unpurified GUS resolved with SDS-PAGE. (D) Calculation of sample purity of the unpurified GUS via integration of the signal intensity of the three sections marked in (C).

cation. The average number of phosphorylation sites per molecule is reported as a ratio of the amount of phosphorylated protein to the amount of total protein (Figure 1D).

To differentiate between phosphate moieties present as phosphorylated glycans and phosphorylated amino acid residues, samples were treated with alkaline phosphatase and/or PNGase $\mathrm{F}$ to remove phosphates and $\mathrm{N}$-linked glycans, respectively. All reactions were performed on 10- $\mu \mathrm{g}$ samples according to the manufacturers' instructions. To minimize variation in enzymatic activity in different buffers, control and experimental samples were treated identically. Briefly, $10 \mu \mathrm{g}$ samples were prepared in alkaline phosphatase buffer (100 $\mathrm{mM}$ glycine, $\mathrm{pH}$ 9.0, $1 \mathrm{mM} \mathrm{MgCl}$, $0.1 \mathrm{mM}$ $\mathrm{ZnCl}_{2}$ ) with $5000 \mathrm{U}$ alkaline phosphatase or water (control and PNGase F-only reactions) in a $10 \mu$ reaction volume. Reactions were performed overnight at $37^{\circ} \mathrm{C}$. Samples were cooled on ice and then $1 \mu \mathrm{l} 10 \times$ PNGase F denaturant buffer was added to each sample, bringing the volume to $11 \mu$ l. The samples were boiled for $10 \mathrm{~min}$ and then prepared according to the manufacturer's protocol for PNGase F treatment. Again, $1 \mu$ water was used in place of enzyme for control and alkaline phosphatase-only treatments. To stop the reactions, $9 \mu \mathrm{l} 4 \times$ Laemmli sample buffer was added, and samples were boiled for 10 min then separated on a $4 \%-20 \%$ TGX precast gel under denaturing conditions. The gel was stained sequentially with Phos-Tag, eLuminol, and PageBlue, then imaged with an E-Gel Imager system after each stain (Figure 1A), as described above.

The PageBlue-stained gel was used to determine the apparent molecular weight of the glycosylated and/or phosphorylated (control), dephosphorylated (treated with alkaline phosphatase), and deglycosylated (treated with PNGase F) protein samples (Figure 1A, bottom). Using the relative migration distance (Rf) value of each of the molecular weight standard (Precision Plus
Protein Dual Color Standards; Bio-Rad); a standard curve was generated, and an apparent molecular weight $(\mathrm{kD})$ was determined to verify the size shift resulting from deglycosylation (Figure 1A, bottom values).

\section{6-well plate assay}

Determination of the number of phosphate groups present on cathepsin D, purified GUS, or dialyzed unpurified GUS was performed in a 96-well plate format. A standard curve for ovalbumin (range: $0.001 \mu \mathrm{g}-100 \mu \mathrm{g}$ ) was generated in duplicate in a final volume of $37.5 \mu \mathrm{l}$ per well. Protein samples $(0.5-60 \mu \mathrm{g}$ per well) were diluted to a final volume of 37.5 $\mu \mathrm{l}$ in water and run in triplicate. We deliberately included amounts of protein outside the linear range of detection to provide a baseline and saturation. To immobilize the protein sample within the well, $37.5 \mu$ melted $2 \%$ agarose (in water) was added to the protein sample at approximately $65-75^{\circ} \mathrm{C}$, mixed carefully (avoiding air bubbles). and then allowed to solidify at room temperature. Phos-Tag (100 $\mu$ l) was added to each well and allowed to incubate for 90 min with gentle agitation. The stain was removed by decanting the plate, and $100 \mu \mathrm{l}$ Phos-Tag Destain (GeneCopoeia) was added to each well and the plate was incubated for 30-45 min (with gentle agitation). The destain was replaced with fresh destain solution and the fluorescence signal was determined with a plate reader (BioTek Synergy H1 Multi-Mode Reader; BioTek Instruments Inc, Winooski, VT) (excitation/emission: 550/580 nm). Phos-Tag Destain was then removed, and $100 \mu \mathrm{l}$ eLuminol was added to each well, followed by a 90 min incubation. The stain was replaced with $100 \mu \mathrm{l}$ eLuminol Destain (GeneCopoeia) and incubated for 30-45 $\mathrm{min}$. The destain was replaced, and the plate was read in the same plate reader (excitation/ emission: 460/600 nm).

Fluorescence intensities obtained from the 96-well-plate method were used to generate a standard curve from the ovalbumin standards stained with both eLuminol (Figure 2A) and Phos-Tag (Figure 2B). Ovalbumin signal from the Phos-Tag was divided by two to account for the two phosphorylation sites. The GUS and cathepsin D fluorescence intensities that were within the linear portion of the standard curve were used to determine the amount of total protein and phosphorylated protein. Relative level of phosphorylation ( $\mathrm{mol} / \mathrm{mol}$ ) was expressed as a ratio of amount of phosphorylated protein to the amount of 


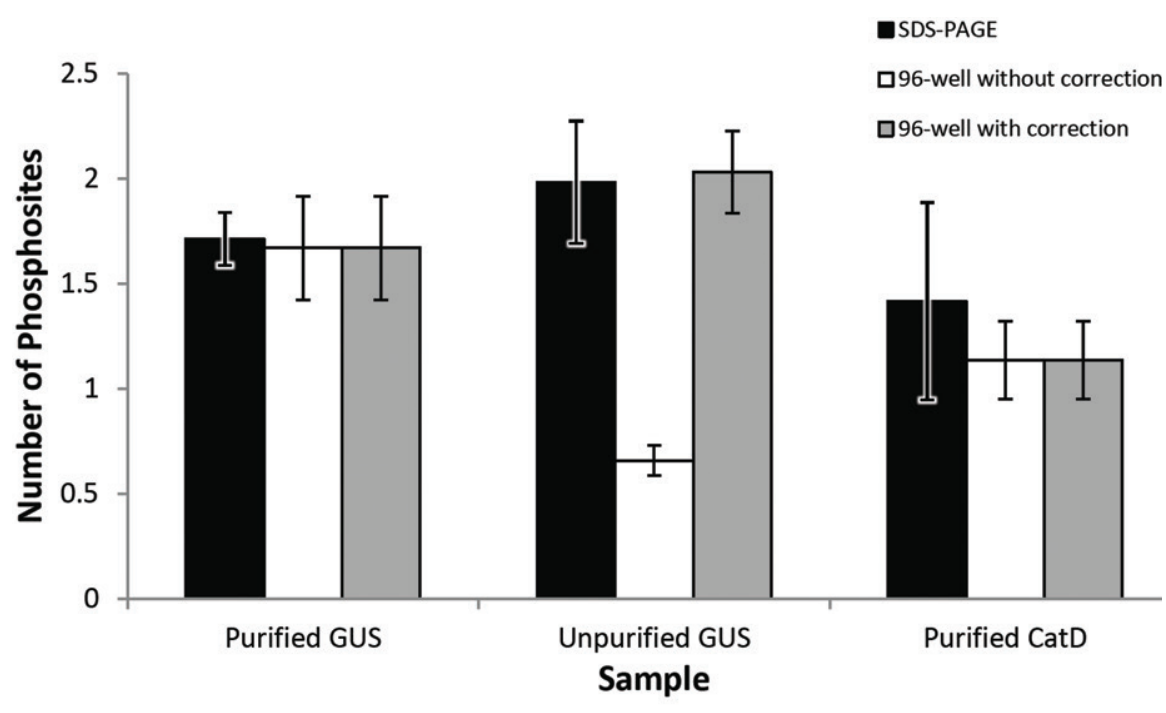

Figure 3. Quantification of the number of phosphorylation sites per molecule. The number of phosphorylation sites per molecule of purified $\beta$-glucuronidase (GUS), dialyzed bioreactor media containing GUS, and commercially obtained purified cathepsin D (CatD) as calculated by the SDS-PAGE method (black bars), 96 well plate method (white bars), and 96-well plate method with correction for sample purity (gray bars). Note the deviation in the determined value of phosphorylation sites per molecule for the unpurified GUS sample when analyzed with the 96-well plate method without correction compared with either the SDS-PAGE or corrected 96-well plate methods. Error bars represent standard error ( $n \geq 7$ for each measurement).

total protein. This method was found to be not suitable for direct quantification of unpurified GUS samples (compare Figure 3, white bars). Prior knowledge of the relative purity of the samples is essential for estimating the relative amount of phosphorylation in these unpurified samples. To determine sample purity, a
PageBlue-stained TGX precast gel with two dilutions of each GUS sample was run. Analysis was performed using GelQuant Express Analysis Software; each lane was divided into three sections (Figure 2C). The first contained all protein bands above the GUS band, the second contained the GUS band, and the third contained all protein bands below the GUS band. The signal intensity was integrated in each of the three sections; the integrated intensity of the GUS band was divided by the sum of the integrated intensities of the entire lane, producing the fractional amount of the total protein consisting of GUS, which is expressed as percent purity. The percent purity was then used to correct the eLuminol-staining measurements, yielding the amount of GUS present per well (Figure 2D), which was comparable to the amount of protein independently determined by a capillary electrophoresis method previously reported (16). Finally, to determine the average number of phosphorylation sites, the calculated amount of phosphorylated protein was divided by the corrected amount of GUS protein to determine the number of phosphates per molecule (Figure 3, gray bars).
Nanoject III The Smallest Big Deal in Microinjection
Completely programmable nanoliter injection control expands your capabilities

- User-friendly touchscreen setup for single injections as well as multiple injection cycle recipes

- Advanced hydraulics deliver precise, consistent injection volumes down to 0.6 nanoliters

- Improved chuck design provides simple, secure micropipet attachment without the need for O-rings

Go to injection.expert for more details about Nanoject III expanded microinjection capabilities.

Nanoject III.. a Big Deal in Microinjection
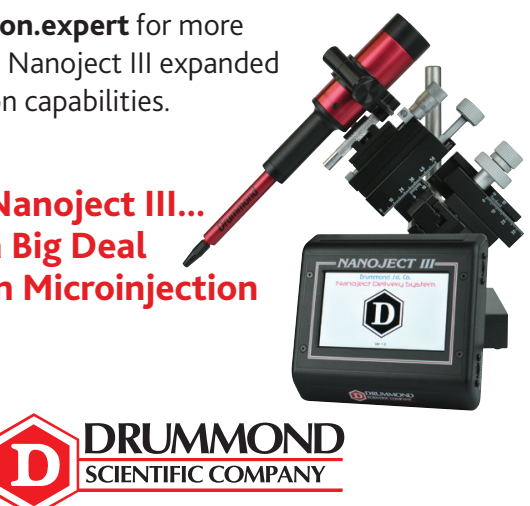

Developers of the original Pipet-Aid ${ }^{\circledR}$

500 Parkway, Box $700 \bullet$ Broomall, PA $19008 \bullet 800.523 .7480$ 


\section{Results and discussion}

The adaptation of selective staining of phosphate molecules by Phos-Tag dye and total protein staining by eLuminol dye has led to the determination of the amount of phosphorylated protein and total protein, respectively. Using these quantities, it is possible to determine relative levels of phosphorylation as the average number of phosphates per molecule. For GUS and cathepsin $D$, two lysosomal enzymes known to possess M6P on their glycans, the results were comparable with published data (14,15,21-26). By employing alkaline phosphatase and PNGase F treatments, it is possible to determine both the average number of phosphate moieties as well as the type of phosphorylation, whether side-chain or glycan. The ovalbumin sample showed that PNGase F treatment results in a size shift consistent with $\mathrm{N}$-linked glycosylation, but does not alter staining with Phos-Tag (Figure 1A), which is consistent with the two phosphate moieties being present on serines 69 and 345 (18,19). Furthermore, treatment of either purified or dialyzed spent media containing GUS with either alkaline phosphatase or PNGase $F$ resulted in a loss of signal for Phos-Tag staining, in agreement with published data indicating that the phosphate moieties are present as phosphorylated glycans (as M6P), rather than as phosphorylated amino acid residues (Figure 1A) (21-26). Likewise, treatment of cathepsin $D$ with either alkaline phosphatase or PNGase F resulted in a loss of signal with Phos-Tag staining, again indicating that the phosphate is present as a phosphoglycan. Furthermore, the analysis of unpurified GUS samples indicates that this method is applicable for analysis of lysosomal enzyme proteins during various stages of production with different levels of purity.

The SDS-PAGE method for analyzing phosphate content with Phos-Tag Phosphoprotein Gel Stain and eLuminol Protein Gel Stain is direct, but it involves sequential staining and destaining with analyses of the gel images. The 96-well plate assay, on the other hand, does not involve image analysis, and it is relatively faster. However, there are caveats to using this methodology. Primarily, it works best with purified protein, but it can be applied to samples containing contaminants, provided information regarding the sample purity is available. Although a correction can be made for an impure sample, it becomes more complicated if any impurities contain phosphate. These impurities will increase the Phos-Tag signal, requiring measurement correction as well. Thus, unpurified samples are better analyzed by the SDS-PAGE method. Additionally, it is not possible to perform either alkaline phosphatase or PNGase F treatment and then perform the 96-well plate format assay to determine the type of phosphorylation. Alkaline phosphatase and PNGase F and their reaction buffers contain phosphate that confound the phosphorylation signal with significant background signal when stained with Phos-Tag. Finally, samples cannot be prepared in a buffer containing phosphate, as we found that a dilution series of PBS significantly interferes with the Phos-Tag fluorescence signal obtained from ovalbumin (data not shown). Given that the linear range for ovalbumin stained with Phos-Tag was 0.05-2.5 nmol (1-40 $\mu \mathrm{g}$ of ovalbumin), any sample prepared in a phosphatecontaining buffer would require significant dilution, which would likely bring the protein concentration below the detection limit of the eLuminol Stain. Thus, these samples are more suitable for analysis with the SDS-PAGE method.

Despite these caveats, the 96-well plate method produced results for purified cathepsin D and GUS samples that are comparable to those of SDS-PAGE method (Figure 3, white bars). Unpurified GUS samples gave protein quantities that were artificially high due to contaminants, resulting in significantly decreased values for the phosphate per molecule (Figure 3, white bars); however, applying the described correction for sample purity (Figure 2, C and D) gave values in agreement with the SDS-PAGE method and published data (Figure 3, gray bars).

The two methods described here allow for analysis of phosphate moieties in many different samples without the need for specialized equipment or technical skills. Not only does our SDS-PAGE method appear suitable for quantifying the number of phosphate moieties, but the selective enzymatic removal of phosphate (alkaline phosphatase) or glycans (PNGase F) allows for determi- nation of the type of phosphate moiety (whether it is present on amino acid residues or on glycans). We demonstrated the utility of this method to assess the relative levels of glycan phosphorylation, likely M6P in the case of these two lysosomal enzymes, cathepsin D (26) and GUS (14,15). This method could also be a valuable tool for analyzing posttranslational phosphorylation state of a multitude of proteins, especially while developing processes for potential biotherapeutics or examining the effects of process variability.

\section{Author contributions}

S.A.K. performed the experiments and wrote the manuscript. M.A. wrote the manuscript. C.N.M. conceptualized the work, performed experiments, and wrote the manuscript.

\section{Acknowledgments}

This study was supported by a CDER intramural research grant for "Product Quality and Biopharmaceutics of Complex Dosage Forms." S.A.K. acknowledges an ORISE fellowship from CDER and the FDA. The authors thank Massod Rahimi and Swamy Tripurani of the Office of Biotechnology Products/CDER for critical reading and comments on the manuscript. This article reflects the views of the authors and should not be construed to represent FDA's views or policies

\section{Competing interests}

The authors declare no competing interests.

\section{References}

1. Fisher, D., L. Krasinska, D. Coudreuse, and B. Novak. 2012. Phosphorylation network dynamics in the control of cell cycle transitions. J. Cell Sci. 125:4703-4711.

2. Shen, T. and S. Huang. 2012. The role of Cdc25A in the regulation of cell proliferation and apoptosis. Anticancer. Agents Med. Chem. 12:631-639.

3. Sur, S. and D.K. Agrawal. 2016. Phosphatases and kinases regulating CDC25 activity in the cell cycle: clinical implications of CDC25 overexpression and potential treatment strategies. Mol. Cell. Biochem. 416:33-46.

4. Solá, R.J. and K. Griebenow. 2009. Effects of glycosylation on the stability of protein pharmaceuticals. J. Pharm. Sci. 98:1223-1245.

5. Solá, R.J. and K. Griebenow. 2010. Glycosylation of therapeutic proteins: an effective strategy to optimize efficacy. BioDrugs 24:9-21. 
6. Mihov, D. and M. Spiess. 2015. Glycosaminoglycans: Sorting determinants in intracellular protein traffic. Int. J. Biochem. Cell Biol. 68:87-91.

7. Potelle, S., A. Klein, and F. Foulquier. 2015. Golgi post-translational modifications and associated diseases. J. Inherit. Metab. Dis. 38:741-751.

8. Parenti, G., G. Andria, and A. Ballabio. 2015. Lysosomal storage diseases: from pathophysiology to therapy. Annu. Rev. Med. 66:471-486.

9. Pereira, C.S., H. Ribeiro, and M.F. Macedo. 2017. From Lysosomal Storage Diseases to NKT Cell Activation and Back. Int. J. Mol. Sci. 18:502-515.

10. Segatori, L. 2014. Impairment of homeostasis in lysosomal storage disorders. IUBMB Life 66:472-477.

11. Staretz-Chacham, O., T.C. Lang, M.E. LaMarca, D. Krasnewich, and E. Sidransky. 2009. Lysosomal storage disorders in the newborn. Pediatrics 123:1191-1207.

12. Meikle, P.J., J.J. Hopwood, A.E. Clague, and W.F. Carey. 1999. Prevalence of Lysosomal Storage Disorders. JAMA 281:249-254.

13. Kinoshita, E., E. Kinoshita-Kikuta, K. Takiyama, and T. Koike. 2006 Phosphate-binding tag, a new tool to visualize phosphorylated proteins. Mol. Cell. Proteomics 5:749-757.

14. Baldwin, E.T., T.N. Bhat, S. Gulnik, M.V. Hosur, R.C. Sowder, R.E. Cachau, J. Collins, A.M. Silva, and J.W. Erickson. 1993. Crystal structures of native and inhibited forms of human cathepsin D implications for lysosomal targeting and drug design. Proc. Natl. Acad. Sci. USA 90:6796-6800.

15. Bones, J., S. Mittermayr, N. McLoughlin, M. Hilliard, K. Wynne, G.R. Johnson, J.H. Grubb, W.S. Sly, and P.M. Rudd. 2011. Identification of $\mathrm{N}$-glycans displaying mannose-6-phosphate and their site of attachment on therapeutic enzymes for lysosomal storage disorder treatment. Anal. Chem. 83:5344-5352

16. Madhavarao, C.N., C.D. Agarabi, L. Wong, S. Muller-Loennies, T. Braulke, M. Khan, H. Anderson, and G.R. Johnson. 2014. Evaluation of butyrateinduced production of a mannose-6-phosphorylated therapeutic enzyme using parallel bioreactors. Biotechnol. Appl. Biochem. 61:184-192.

17. Grubb, J.H., C. Vogler, B. Levy, N. Galvin, Y. Tan, and W.S. Sly. 2008. Chemically modified beta-glucuronidase crosses blood-brain barrier and clears neuronal storage in murine mucopolysaccharidosis VII. Proc. Natl. Acad. Sci. USA 105:2616-2621.

18. Henderson, J.Y., A.J.G. Moir, L.A. Fothergill, and J.E. Fothergill. 1981. Sequences of sixteen phosphoserine peptides from ovalbumins of eight species. Eur. J. Biochem. 114:439-450.

19. Stein, P.E., A.G.W. Leslie, J.T. Finch, and R.W. Carrell. 1991. Crystal structure of uncleaved ovalbumin at 1.95 ̊ resolution. J. Mol. Biol. 221:941959.

20. Nisbet, A.D., R.H. Saundry, A.J.G. Moir, L.A. Fothergill, and J.E. Fothergill. 1981. The Complete Amino- Acid Sequence of Hen Ovalbumin. Eur. J. Biochem. 115:335-345.

21. Chen, R., X. Jiang, D. Sun, G. Han, F. Wang, M. Ye, L. Wang, and H. Zou. 2009. Glycoproteomics Analysis of Human Liver Tissue by Combination of Multiple Enzyme Digestion and Hydrazide Chemistry. J. Proteome Res. 8:651-661.

22. Freeze, H.H. and C. Kranz. 2008. Endoglycosidase and glycoamidase release of $\mathrm{N}$-linked glycans. Curr Protoc Immunol. Chapter 8:Unit 8.15.

23. Halim, A., U. Ruetschi, G. Larson, and J. Nilsson. 2013. LC-MS/MS characterization of O-glycosylation sites and glycan structures of human cerebrospinal fluid glycoproteins. J. Proteome Res. 12:573-584.

24. Lewandrowski, U., J. Moebius, U. Walters, and A. Sickmann. 2006. Elucidation of $\mathrm{N}$-glycosylation sites on human platelet proteins a glycoproteomic approach. Mol. Cell. Proteomics 5:226-233.

25. Liu, T., W.J. Qian, M.A. Gritsenko, D.G. Camp, M.E. Monroe, R.J. Moore, and R.D. Smith. 2005. Human Plasma N-Glycoproteome Analysis by Immunoaffinity. J. Proteome Res. 4:2070-2080.

26. Zhang, H., X.J. Li, D.B. Martin, and R. Aebersold. 2003. Identification and quantification of $\mathrm{N}$-linked glycoproteins using hydrazide chemistry, stable isotope labeling and mass spectrometry. Nat. Biotechnol. 21:660-666.

Received 01 June 2017; accepted 11 July 2017

Address correspondence to Chikkathur N. Madhavarao, Division of Product Quality Research, Office of Testing and Research, Office of Pharmaceutical Quality, CDER, FDA, 10903 New Hampshire Ave., Silver Spring, MD 20993. Email: chikkathur.madhavarao@fda.hhs.gov

\section{STATE OF THE ART SYSTEM FOR GEL AND BLOT IMAGING}

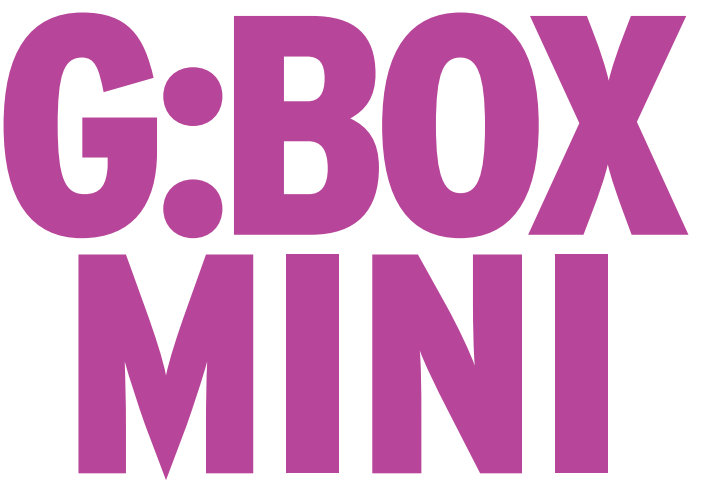

\section{FOR ALL YOUR APPLICATION NEEDS}

Suitable for chemiluminescence, fluorescence, DNA, multiplexing western blots or gels

$\checkmark$ Faster imaging with HI-LEDs to improve workflow
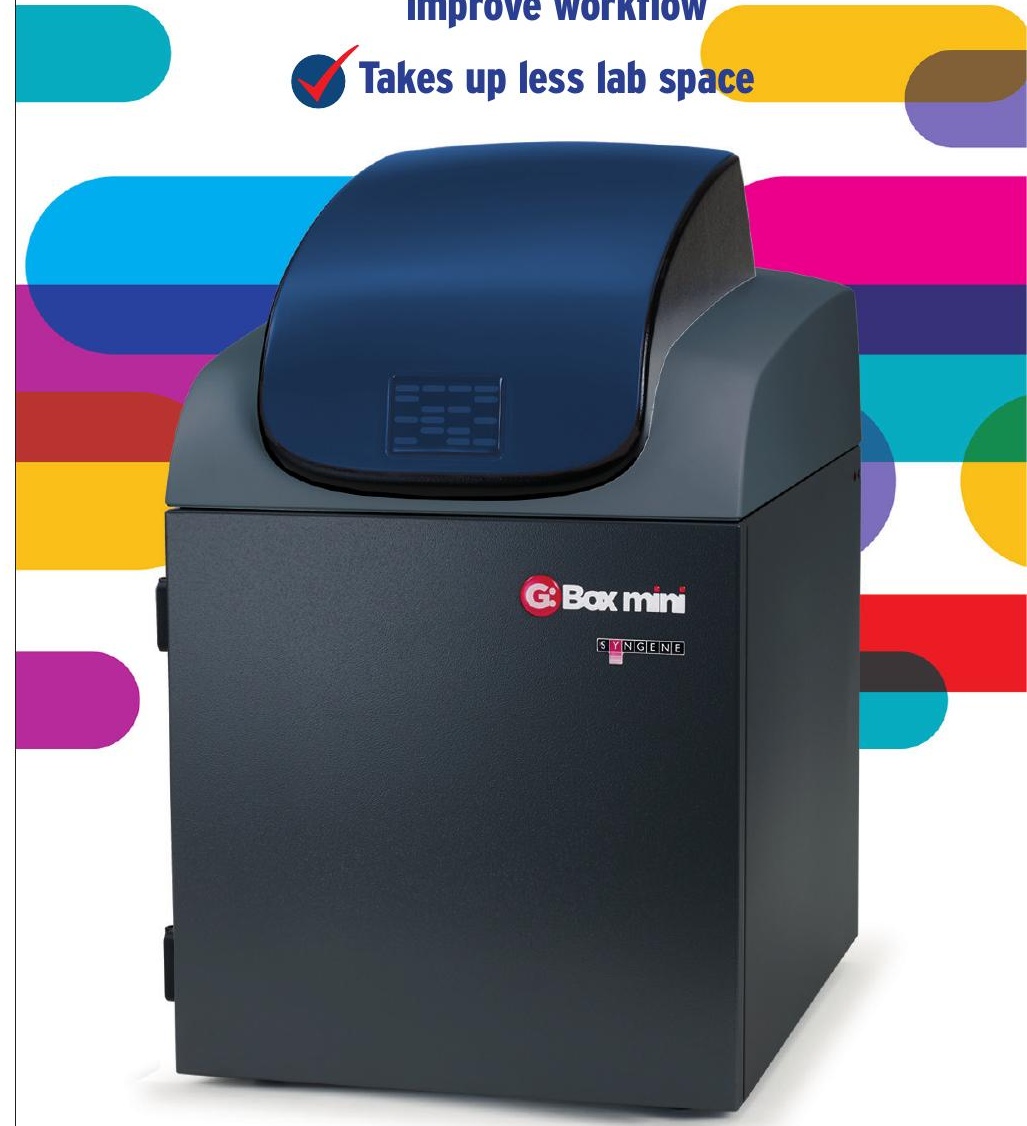

For further information, please contact ussales@syngene.com www.syngene.com/gbox-mini-gel-doc 